sary before construction could be envisaged. The study group was charged with the task of defining the basic problems and establishing the necessary R. \& D. programme. Three main areas of concern have been identified: stability of the magnet with respect to perturbations, fabrication of the conductor and impregnation of the coils.

A detailed plan is being worked out for comparing proven techniques and possible extensions. The conductor should carry $20 \mathrm{kA}$ in fields up to $4 \mathrm{~T}$. With up to $70 \mathrm{~km}$ of conductor required for one magnet, its cost represents a large fraction of the total cost. It is anticipated that some two years of intensive R. \& D. work in close collaboration with industry will be required before the final specifications can be established.

\section{Mathematica $^{\text {тм }}$}

A System for Doing Mathematics by Computer

\section{A Wolfram Research Inc. product}

$\square$ Numerics - Works with numbers of arbitrary magnitude and precision.

$\square$ Symbolics - Encyclopaedia of mathematical functions and operations used in arithmetic, algebra and analysis.

Procedural, functional and mathematical programming.

$\square$ Graphics - 2D, 3D and animated PostScript graphics.

Text processing - Fully interactive reports and textbooks.

$\square$ Runs on - MS-DOS based computers; Macintosh, Apollo, Hewlett Packard, IBM AIX/RT, MIPS, Silicon Graphics, Sony, Sun, VAX.

Now available in Europe from:

MathSoft Overseas, Inc.

POB 641, 1211 Geneva 3, Switzerland

Tel. ++41 (22) 465260

Fax $++41(22) 465939$

\title{
Particle Detectors and Industry
}

The experimental challenge at LHC calling for a close collaboration between the particle physics community and industry promises important spin-offs.

New frontiers in particle physics could historically only be reached by combining progress in accelerator technology with that of experimental techniques, including detectors, electronics, data acquisition, and analysis. Experiments had to become not only larger and more rapid but also much more complex and sophisticated: they could only be constructed and operated by large international collaborations.

Given the need to develop international cooperation and to handle enormous amounts of data, it is no surprise that the particle physics community vigorously promotes broadband data communication systems as well as open (interconnectable) multi-vendor computer networks. The same philosophy of open systems and networking applies to distributed computer-aided design and engineering whereby design teams in several locations can contribute simultanously to projects. The community also remains a test-bench for frontier developments in computing as it requires groups of computers operating in parallel ("farms") and machines based on massively parallel processing for large simulations.

A LHC experiment will contain many millions of read-out channels, each corresponding to the requirements of a high-definition television receiver. Both will require similar high-quality components so the incentive for a close interaction with industry leading to low-cost production is clear. High energy physics will again offer a thorough evaluation of performance limitations.

Owing to the need for rapid data acquisition, particle physics initiated new international communication standards such as NIM, Camac and FASTBUS, where the first two subsequently became widely used in industry. The community is presently participating in the development of a new generation of even faster bus systems for data. Trigger formation will call for a very flexible cross-linking of large data volumes at high rates based on integrating several industrial techniques only now entering development.

\section{Full Professor in Theoretical Physics Technische Universität Graz, Austria}

The tenured position of a Full Professor in Theoretical Physics is available at the Institut für Theoretische Physik, Technisch-Naturwissenschaftliche Fakultät, Technische Universität Graz, Austria. The male/female candidate should be an individual with proven capability to do original research in Many Particle Theory with applications to Condensed Matter Physics. Teaching will include the full curriculum of Theoretical Physics and will be conducted in collaboration with other university teachers of the institute. The applicant should have a Ph.D. in physics and the venia legendi in Theoretical Physics or an equivalent qualification. Applications with curriculum vitae, list of publications, description of research activities, a survey of the teaching experience, and a few essential publications showing the main research interests should be sent to the Dekan der Technisch-Naturwissenschaftlichen Fakultät, Technische Universität Graz, Petersgasse 16, A-8010 Graz, Austria by 31 July 1992. Phone: +43 (316) 8738110 . Female Candidates are especially encouraged to apply.

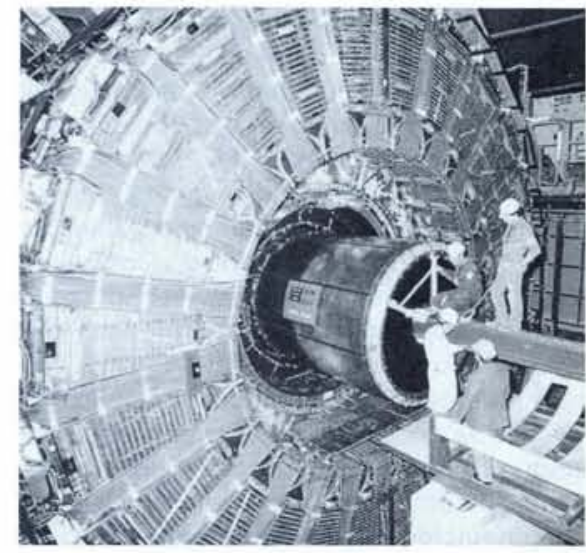

The DELPHI detector at LEP.

For the detector magnets, after initial problems, European industry managed to meet the tight tolerances and to be competitive in producing the superconducting cables required for two LEP experiments. The higher performance requirements for the LHC experiments magnets clearly represent a new challenge.

Regarding detector components, many new techniques have been invented by particle physics groups during the past decades, often relying on special industrial products (composites, high purity quartz, conductive plastics) and some finding various other applications (wire chambers of all kinds, new scintillators, etc.). This development activity continues and has indeed been stimulated immensely by LHC's stringent demands. Take the case of scintillating crystals: emerging requirements have brought together a large group of research institutes and companies involved in crystal growth - the former mainly evaluate performance, while the latter contribute their experience in mass production. These crystals find important applications outside particle physics owing to their compactness, fast response and high resolution.

So too for silicon detectors. The particle physics community initiated a broad line of developments in industry, both for the detectors and the associated electronics which requires an ever-increasing degree of large-scale integration. Many applications are on the horizon, including simple versions of recent pixel devices that have already entered X-ray research and astronomy. The next generation will permit readouts several orders of magnitude faster than today's CCD's used in video cameras.

These few examples illustrate that the interaction with industry proceeds on three levels. Particle physics often acts as a normal consumer of more-or-less standard products. In many cases, however, it participates actively in the developments of new products with exceptional performance. Finally, it sometimes invents techniques which find extensive use in other fields. 